\title{
When is it Better to Wait for a New Version? Optimal Replacement of an Emerging Technology under Uncertainty
}

\author{
Michail Chronopoulos • Afzal Siddiqui
}

Received: date / Accepted: date

\begin{abstract}
Firms that use an emerging technology often face uncertainty in both the arrival of new versions and the revenue that may be earned from their deployment. Via a sequential decision-making framework, we determine the value of the investment opportunity and the optimal replacement rule under three different strategies: compulsive, laggard, and leapfrog. In the first one, a firm invests sequentially in every version that becomes available, whereas in the second and third ones, it first waits for a new version to arrive and then either invests in the older or the newer version, respectively. We show that, under a compulsive strategy, technological uncertainty has a non-monotonic impact on the optimal investment decision. In fact, uncertainty regarding the availability of future versions may actually hasten investment. By comparing the relative values of the three strategies, we find that, under a low output price the compulsive strategy always dominates, whereas, at a high output price, the incentive to wait for a new version and adopt either a leapfrog or a laggard strategy increases as the rate of innovation increases. By contrast, high price uncertainty mitigates this effect, thereby increasing the relative attraction of a compulsive strategy.
\end{abstract}

Keywords investment analysis $\cdot$ real options $\cdot$ emerging technologies $\cdot$ dynamic programming

\section{Introduction}

A distinctive feature of emerging technologies is the rapid pace of innovation, which together with uncertainty in revenue streams in deregulated industries pose a formidable challenge for firms devising strategies for technology replacement. For example, government subsidies for renewable energy (RE) technologies have fuelled a boom in wind turbine manufacturing. However, although wind turbine replacement is considered a more attractive alternative compared to investing in a new wind farm (Wind Power, 2012), it is, nevertheless, considerably

Michail Chronopoulos

Department of Business and Management Science, Norwegian School of Economics, Bergen 5045, Norway

Tel.: +4755959385

E-mail: Michail.Chronopoulos@nhh.no

Afzal Siddiqui

Department of Statistical Science, University College London, London WC1E 6BT, UK

Department of Computer and Systems Sciences, Stockholm University, Stockholm SE-106 91, Sweden 
capital intensive, and, in an uncertain economic environment, power companies are cautious when determining which turbine version to adopt and when. Likewise, the automation of services has made most firms reliant on IT systems, yet, considering the high rate at which software is updated by providers (Financial Times, 2012a), users are faced with the problem of timely adoption of the correct version. In particular, how should a firm adopt new versions of an existing technology when it faces both economic and technological uncertainty? Are there situations in which it would be optimal for the firm to skip adopting an available version and to wait for a newer model of the technology? What are the policy implications when firms use such adoption strategies? We take a real options approach (Dixit and Pindyck, 1994) in order to compare technology-adoption strategies under uncertainty. By allowing for both economic and technological uncertainty, it is possible to analyse how these interact, and, thus, uncover the dynamics that determine the dominant technology-adoption strategy in different regimes. The novelty of our work is that it facilitates the derivation of analytical results regarding the impact of price and technological uncertainty on both the feasibility of technology-adoption strategies and the associated investment policies.

Although both technological decay and the random arrival of innovations reflect technological uncertainty, empirical evidence has shown that the latter presents a greater incentive for replacing a technology. For example, in the computer industry, hardware and software companies often design a new version so that the value of an earlier one is reduced. As a result, private investors replace equipment typically due to planned obsolescence and not because its lifetime has expired (The Economist, 2009). This has far-reaching consequences considering that many industries rely gradually more on computer technologies, and, thus, private investors are faced with the task of making timely investment and operational decisions under increasing technological uncertainty. Similarly, in the area of wind turbines, empirical research has indicated that innovation, rather than technological decay, is the primary cause of turbine replacement (Jensen et al., 2002). Of course, the replacement of a RE technology may be also influenced by other factors that are beyond the scope of this paper, e.g., the limited availability of resource-rich locations. For example, the opportunity cost from delaying the replacement of wind turbines in wind-rich locations is endogenously related to the availability of land. Consequently, empirical analysis has indicated that policies that are implemented in order to encourage the scrapping of older, poorly placed turbines are inefficient as they have a larger effect on turbines located in areas with better wind resources (Mauritzen, 2014).

Furthermore, estimating the profit of projects based on emerging technologies is a complicated process as it typically depends on several factors. For example, in the case of a wind farm, the annual revenue may depend on electricity prices, wind speeds, and feed-in tariffs, as well as other random variables. Nevertheless, in order to enable mathematical tractability, investment models usually address such features separately, and, as a result, questions regarding their combined impact on investment and operational decisions remain open. In this paper, we address this disconnect by developing a real options framework in order to tackle the problem of optimal replacement of a technology under output price and technological uncertainty. As a result, the 
contribution of this paper is threefold. First, we develop an analytical framework for sequential replacement of an emerging technology. Second, we derive analytical and numerical results on the effect of price and technological uncertainty as well as their interaction on the decisions to upgrade a technology by replacing old equipment with more efficient versions. Third, we provide managerial insights for replacement decisions based on analytical and numerical results. Specifically, we show that price and technological uncertainty interact to affect the optimal technology-adoption strategy when the output price is high and that this decision is independent of technological uncertainty when the output price is low. The impact of this tradeoff is evident in various industries, e.g., RE and telecommunications. In the former, investors refrained from adopting new wind turbines in 2008 due to increasing economic uncertainty, although Vestas continued to invest in R\&D (Financial Times, $2012 \mathrm{~b}$ ), whereas, in the latter, the impending release of iPhone 6 increased consumers' incentive to ignore previous versions (International Business Times, 2014).

We proceed by discussing some related work in Section 2 and introduce assumptions and notation in Section 3. We address the problem of exercising a single replacement option in Section 4 and analyse a compulsive strategy, where a firm adopts two subsequent technologies, in Section 5. In Section 6, we analyse the case where a firm can adopt either a leapfrog or a laggard strategy, and in Section 7, we compare these two strategies with the compulsive one and show how the optimal strategy can be determined endogenously. Section 8 provides numerical examples for each case and illustrates the interaction between price and technological uncertainty in order to enable more informed replacement decisions. Section 9 concludes and offers directions for future research.

\section{Related Work}

Despite the significant literature in the area of sequential investment, analytical formulations of problems that combine price and technological uncertainty are limited. Early examples in the area of sequential investment include Majd and Pindyck (1987), who show how traditional valuation methods understate the value of a project by ignoring the flexibility embedded in the time to build. Dixit and Pindyck (1994) develop an analytical framework for sequential investment assuming that the output price follows a geometric Brownian motion (GBM), the project value depreciates exponentially, and the investor has an infinite set of replacement options. More recent examples include Gollier et al. (2005), who compare a flexible sequence of small nuclear power plants with a single nuclear power plant of large capacity and show that the value of modularity may even trigger investment in the initial module at an electricity price level below the now-or-never NPV threshold. Malchow-Møller and Thorsen (2005) illustrate that, due to the possibility of updating equipment when investing in an alternative energy technology, the required investment threshold is less sensitive to changes in uncertainty and resembles the investment behaviour under the simple NPV rule. Kort et al. (2010) compare a single-stage investment to a flexible stepwise investment strategy and show that higher price uncertainty makes the former strategy more attractive relative to the latter by raising the reluctance to make costly switches between states. 
In the area of investment under technological uncertainty, Balcer and Lippman (1984) analyse the optimal timing of technology adoption under infinite switching options and find that the timing of technology adoption is influenced by expectations about future technological changes and that increasing technological uncertainty tends to delay adoption. Grenadier and Weiss (1997) develop a model for sequential investment, whereby a firm may either adopt every technology that becomes available (compulsive), skip an old technology in order to adopt the next one (leapfrog), purchase only an early innovation (buy and hold), or wait for a new technology to arrive before adopting the previous one (laggard). In each case, they illustrate how the rate of innovation and technological growth impact the optimal technology-adoption strategy and find that a firm may adopt an available technology even though more valuable innovations may occur in the future. Nevertheless, how price and technological uncertainty interact to affect the optimal investment rule under each strategy is not thoroughly discussed.

Farzin et al. (1998) investigate the optimal timing of technology adoption assuming that new technologies arrive according to a Poisson process, however, they consider a deterministic production function, and, thus, ignore output price uncertainty. Doraszelski (2001) identifies an error in Farzin et al. (1998) and shows that, compared to the NPV approach, a firm will defer the adoption of a new technology when it takes the option value of waiting into account. Huisman and Kort (2004) analyse a duopoly in which firms face price and technological uncertainty and show that the efficiency of a new technology can offset the monopoly profits that a leader receives while being alone in the market, thereby turning a pre-emption game into a war of attrition. Kauffman and Li (2005) use a standard Brownian motion in order to describe uncertainty in the outcome of technology competition and analyse the investment strategy of a firm that can choose between two competing technologies. Miltersen and Schwartz (2007) adopt a real options approach for valuing R\&D projects under uncertain time to completion, operational flexibility, and competition. Franklin (2014) proposes a real options model for valuing the option to invest in a mobile telecommunications network under price and technological uncertainty. Assuming that the latter reflects the exponential decay of network elements, he analyses the case in which a mobile operator has a single investment option as well as the option to replace a network element once the project dies.

An implication of technological uncertainty is that a firm may have to choose between alternative projects. Dixit (1993) analyses an irreversible choice among mutually exclusive projects under output price uncertainty and finds that increasing returns and uncertainty make it optimal to wait for the largest project. Décamps et al. (2006) extend Dixit (1993) by providing parameter restrictions under which the optimal investment strategy is not a trigger strategy and the optimal investment region is dichotomous. Siddiqui and Fleten (2010) analyse how a firm may proceed with staged commercialisation and deployment of competing alternative energy technologies. They consider a setting in which a firm can choose between a new alternative technology, which requires cost-reducing enhancement measures prior to deployment, and an existing RE technology. Although 
these are examples of analytical frameworks for investment in alternative projects, the availability of these projects is taken for granted as it is not subject to a probability distribution.

In this paper, we develop a framework for sequential investment in which we analyse the tradeoff between continuing to run an old technology and replacing it with successively improved versions under price and technological uncertainty. Therefore, we analyse three strategies, i.e., compulsive, leapfrog, and laggard; however, we deviate from the framework of Grenadier and Weiss (1997) by analysing their endogenous relation assuming a stochastic price process that facilitates the analysis of the impact of price and technological uncertainty on the optimal investment rule under each strategy. Thus, we derive more detailed insights on how technologyadoption strategies depend on price and technological uncertainty and how these features impact the value of a project and the optimal investment rule. We show that, under a compulsive strategy, technological uncertainty has a non-monotonic impact on the optimal investment decision and may actually accelerate investment. Additionally, we determine the range of prices in which the optimal strategy depends on technological uncertainty and find that a high output price decreases the required rate of innovation for which a firm is willing to ignore an available technology until a new one becomes available so that it has the option to choose between the two. Interestingly, high price uncertainty mitigates this effect by decreasing the feasibility of a laggard strategy and increasing the likelihood of a downturn, which, in turn, reduces the attractiveness of the new technology.

\section{Assumptions and Notation}

We assume that a price-taking firm has options to adopt $n=1,2,3, \ldots, N$ successively improved versions of an emerging technology with $N<\infty$. Given a probability space $(\Omega, \mathcal{F}, \mathbb{P})$, we assume that innovations associated with the emerging technology follow a Poisson process $\left\{M_{t}, t \geq 0\right\}$, where $t$ is continuous and denotes time. This enables the analysis when firms have no information about the decisions made by R\&D companies. The process $M_{t}$ is defined in (1):

$$
M_{t}=\sum_{k \geq 1} \mathbb{1}_{\left\{t \geq T_{k}\right\}}
$$

where $T_{k}=\sum_{n=1}^{k} y_{n}$ and $\left\{y_{n}, n \geq 1\right\}$ is a sequence of independent and identically distributed random variables, such that $y_{n} \sim \exp (\lambda), \forall n \geq 1$, i.e., $f_{Y}(y)=\lambda e^{-\lambda y_{1}} y \geq 0$. Parameter $\lambda \in \mathbb{R}^{+}$denotes the intensity of the Poisson process and is independent of $t$. Intuitively, $M_{t}$ counts the number of random times $T_{k}$ that occur between 0 and $t$, and $y_{n}=T_{n}-T_{n-1}$ is the time interval between subsequent innovations. Hence, if no innovation has occurred for $t$ years, then, with probability $\lambda d t$, an innovation will occur within the next short interval of time $d t$, i.e.,

$$
d M_{t}= \begin{cases}1 & , \text { with probability } \lambda d t \\ 0 & , \text { with probability } 1-\lambda d t\end{cases}
$$


We assume that there is no operating cost associated with the technology and the output price at time $t$, $E_{t}$, is independent of the Poisson process and follows a GBM that is described in (2), where $\mu$ is the annual growth rate, $\sigma$ is the annual volatility, $d Z_{t}$ is the increment of the standard Brownian motion, and $\rho \geq \mu$ is the subjective discount rate.

$$
d E_{t}=\mu E_{t} d t+\sigma E_{t} d Z_{t}, E_{0} \equiv E>0
$$

The output of technology version $n$ is $D_{n}\left(D_{n+1} \geq D_{n}, \forall n\right)$, and the corresponding investment cost is $I_{n}$. Additionally, $\tau_{\ell, m, n}^{(N)}$, where $\ell, m, n \in \mathbb{N}$, is the time at which version $m$ is adopted given that version $\ell<m$ is installed while version $n, n \geq m>\ell$, is available, and $\epsilon_{\ell, m, n}^{(N)}$ denotes the corresponding optimal adoption threshold price with $N$ total versions available. For example, $\tau_{0,1,2}^{(N)}$ is the optimal time to invest in version 1 when version 2 is the latest one available and no version is currently in operation, while $\epsilon_{0,1,2}^{(N)}$ is the corresponding optimal investment threshold. All options are perpetual, and installed versions last forever. Finally, $F_{\ell, m, n}^{(N)}(\cdot)$ is the maximised expected NPV from investment in version $m$ given that version $\ell$ is in operation and version $n$ is the latest one available for adoption, while $\Phi_{\ell, n}^{(N)}(\cdot)$ is the expected value of a project inclusive of embedded options when version $\ell$ is installed and version $n$ is the latest one available. Note that, in order to have a tradeoff between an old and a more efficient version, we assume that at the point, $\varepsilon$, where the NPVs of the two project are equal, i.e., $\varepsilon$ is such that $\Phi_{\ell, n}^{(N)}(\varepsilon)=\Phi_{n, n}^{(N)}(\varepsilon)$, we have $\Phi_{n, n}^{(N)}(\varepsilon)>0$. Otherwise, only the newer version presents a viable investment opportunity, as its NPV is always greater than that of the old version for all the positive values of its range (Décamps et al., 2006). As we will show in Section 6, this condition implies $\frac{D_{n}}{\sum_{i=1}^{n} I_{i}}<\frac{D_{\ell}}{\sum_{i=1}^{\ell} I_{i}}, \forall n, \ell$ with $\ell<n$. Intuitively, a new version may produce a greater output compared to an old one, yet is much more capital intensive. The relationship between the investment cost and capacity assumes a common arrival rate for all technology versions, yet this could be relaxed to yield heterogeneous rates of technological uncertainty that are linked to the investment cost as well.

\section{Benchmark Case}

The firm holds a single option to invest in a technology that will become available at a random time $T_{1}$. Figure 1 indicates the different states and corresponding value functions. Notice that a transition due to a Poisson event (investment) is indicated by a broken (solid) arrow. In state $(0,0)$, the firm holds an option to invest in a technology that is not available but may arrive according to a Poisson process. When that happens, the firm moves into state $(0,1,1)$ where it can exercise the option by incurring a fixed cost, thus moving to state $(1,1)$ where it continues to operate technology 1 forever.

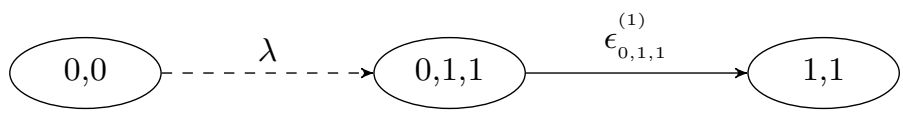

Fig. 1 State transition diagram for $N=1$ 
In order to determine the value function in each state, we use backward induction and first consider state $(1,1)$. When the first technology becomes available, the firm has the option to incur a fixed cost, $I_{1}$, in order to adopt it at time $\tau_{0,1,1}^{(1)}$ and the expected NPV from immediate investment is described in (3).

$$
\Phi_{1,1}^{(1)}(E)=\frac{D_{1} E}{\rho-\mu}-I_{1}
$$

Notice that at $T_{1}$, we can have either $E<\epsilon_{0,1,1}^{(1)}$ or $E \geq \epsilon_{0,1,1}^{(1)}$. Thus, the value of the investment opportunity in state $(0,1,1)$ is described in $(4)$

$$
F_{0,1,1}^{(1)}(E)= \begin{cases}A_{0,1,1}^{(1)} E^{\beta_{1}} & , E<\epsilon_{0,1,1}^{(1)} \\ \Phi_{1,1}^{(1)}(E) & , E \geq \epsilon_{0,1,1}^{(1)}\end{cases}
$$

where $\beta_{1}>1$ and $\beta_{2}<0$ are the roots of $\frac{1}{2} \sigma^{2} \beta(\beta-1)+\mu \beta-\rho=0$ (all proofs can be found in the appendix). The endogenous constant, $A_{0,1,1}^{(1)}$, and the investment threshold, $\epsilon_{0,1,1}^{(1)}$, are determined via the value-matching and smooth-pasting conditions between the two branches of (4) and are indicated in (5).

$$
A_{0,1,1}^{(1)}=\frac{\epsilon_{0,1,1}^{(1)^{1-\beta_{1}}}}{\beta_{1}} \frac{D_{1}}{\rho-\mu} \text { and } \epsilon_{0,1,1}^{(1)}=\frac{\beta_{1}}{\beta_{1}-1} \frac{I_{1}(\rho-\mu)}{D_{1}}
$$

Since there are no embedded investment options to impact the initial investment decision, $\epsilon_{0,1,1}^{(1)}$ is independent of $\lambda$. Hence, a higher $\lambda$ increases the likelihood of an innovation, yet does not affect the optimal investment rule, which is subject to the GBM.

In state $(0,0)$, an innovation has not occurred yet but is likely to occur at some random time, $T_{1}$, in the future. Thus, the value function in $(0,0)$ is described in $(6)$.

$$
\Phi_{0,0}^{(1)}(E)=(1-\rho d t)\left[\lambda d t \mathbb{E}_{E}\left[F_{0,1,1}^{(1)}(E+d E)\right]+(1-\lambda d t) \mathbb{E}_{E}\left[\Phi_{0,0}^{(1)}(E+d E)\right]\right]
$$

The first term on the right-hand side of (6) reflects the value of the option to adopt a technology if it becomes available over the time interval $d t$, while the second term is the value of continuing to wait if an innovation does not take place over the time interval $d t$. By expanding the right-hand side of (6) using Itô's lemma, we can re-write (6) as follows:

$$
\frac{1}{2} \sigma^{2} E^{2} \Phi_{0,0}^{(1)^{\prime \prime}}(E)+\mu E \Phi_{0,0}^{(1)^{\prime}}(E)-(\rho+\lambda) \Phi_{0,0}^{(1)}(E)+\lambda F_{0,1,1}^{(1)}(E)=0
$$

Notice that for $E<\epsilon_{0,1,1}^{(1)}$, even if an innovation takes place, then it cannot be adopted immediately, and $F_{0,1,1}^{(1)}(E)$ is expressed via the top part of $(4)$. Otherwise, the expression for $F_{0,1,1}^{(1)}(E)$ is indicated in the bottom part of (4). The expression for $\Phi_{0,0}^{(1)}(E)$ is indicated in (8), where $A_{0,1,1}^{(1)}$ is described in (5), $\delta_{1} \geq \beta_{2}$ and $\delta_{2} \leq \beta_{2}$ are the roots of the quadratic $\frac{1}{2} \sigma^{2} \delta(\delta-1)+\mu \delta-(\rho+\lambda)=0$, and $A_{0,0}^{(1)}<0$ and $B_{0,0}^{(1)}>0$ are determined via 
the value-matching and smooth-pasting conditions and are indicated in $(\mathrm{A}-3)$ and $(\mathrm{A}-4)$ respectively.

$$
\Phi_{0,0}^{(1)}(E)= \begin{cases}A_{0,1,1}^{(1)} E^{\beta_{1}}+A_{0,0}^{(1)} E^{\delta_{1}} & , E<\epsilon_{0,1,1}^{(1)} \\ \frac{\lambda D_{1} E}{(\rho+\lambda-\mu)(\rho-\mu)}-\frac{\lambda I_{1}}{\rho+\lambda}+B_{0,0}^{(1)} E^{\delta_{2}} & , E \geq \epsilon_{0,1,1}^{(1)}\end{cases}
$$

The first term in the top part of (8) is the option to invest should an innovation arrive; however, since this option is not available yet, we need to adjust the option value via the second term. The first term in the bottom part of (8) reflects the expected present value of the revenues from the new technology, and the second term is the expected investment cost. Finally, the third term reflects the probability that the price will drop into the waiting region before the occurrence of the innovation. The numerator on the left-hand side of (9) reflects the difference between the option to invest in the absence of technological uncertainty, i.e., $F_{0,1,1}^{(1)}(E)=A_{0,1,1}^{(1)} E^{\beta_{1}}$, and the value function in state $(0,0)$. The latter consists of the option to invest reduced by the term $A_{0,0}^{(1)} E^{\delta_{1}}$ which is negative, thereby reflecting the impact of technological uncertainty. Consequently, the ratio of this difference and $F_{0,1,1}^{(1)}(E)$ reflects the relative loss in the value function $\Phi_{0,0}^{(1)}(E)$ due to technological uncertainty.

$$
\frac{F_{0,1,1}^{(1)}(E)-\Phi_{0,0}^{(1)}(E)}{F_{0,1,1}^{(1)}(E)}=-\frac{A_{0,0}^{(1)} E^{\delta_{1}}}{A_{0,1,1}^{(1)} E^{\beta_{1}}}, E<\epsilon_{0,1,1}^{(1)}
$$

Hence, if $\lambda=0$, then no innovation will occur and $\Phi_{0,0}^{(1)}(E)=0$. By contrast, when $\lambda \rightarrow \infty$, the loss in value due to the likelihood of an innovation converges to zero, and, thus, $\Phi_{0,0}^{(1)} \rightarrow F_{0,1,1}^{(1)}$. Consequently, $\Phi_{0,0}^{(1)} \in\left[0, F_{0,1,1}^{(1)}\right)$ $\forall \lambda \in \mathbb{R}^{+}$(see Proposition 1).

\section{Compulsive Strategy}

We extend the previous framework by assuming that a firm holds two investment options and that it invests in each version of the technology that becomes available ignoring the option to wait to choose between the two. Hence, the transition diagram of Figure 1 is extended by adding states $(1,2,2)$ and $(2,2)$ as in Figure 2. While the assumption that a version becomes available only after the adoption of the previous one is somewhat unrealistic, it serves as a benchmark for comparison in the full model of Section 6. Therefore, we assume that the firm chooses the compulsive strategy exogenously ignoring the potential arrival of a new version.

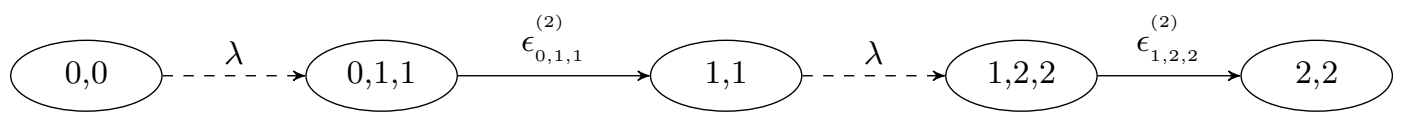

Fig. 2 State transition diagram for $N=2$

The expected NPV from immediate investment in the second version is described in (10).

$$
\Phi_{2,2}^{(2)}(E)=\frac{D_{2} E}{\rho-\mu}-\left(I_{2}+I_{1}\right)
$$


Next, the value function in state $(1,2,2)$ is indicated in $(11)$

$$
F_{1,2,2}^{(2)}(E)= \begin{cases}\Phi_{1,1}^{(1)}(E)+A_{1,2,2}^{(2)} E^{\beta_{1}} & , E<\epsilon_{1,2,2}^{(2)} \\ \Phi_{2,2}^{(2)}(E) & , E \geq \epsilon_{1,2,2}^{(2)}\end{cases}
$$

where the endogenous constant $A_{1,2,2}^{(2)}$ and investment threshold $\epsilon_{1,2,2}^{(2)}$ are indicated in (12).

$$
A_{1,2,2}^{(2)}=\frac{\epsilon_{1,2,2}^{(2)^{1-\beta_{1}}}}{\beta_{1}} \frac{\left(D_{2}-D_{1}\right)}{\rho-\mu} \text { and } \epsilon_{1,2,2}^{(2)}=\frac{\beta_{1}}{\beta_{1}-1} \frac{I_{2}(\rho-\mu)}{D_{2}-D_{1}}
$$

Thus, the value of a project with an installed first technology and a single remaining embedded replacement option, given that the new version is not available yet, is

$$
\Phi_{1,1}^{(2)}(E)= \begin{cases}\Phi_{1,1}^{(1)}(E)+A_{1,2,2}^{(2)} E^{\beta_{1}}+A_{1,1}^{(2)} E^{\delta_{1}} & , E<\epsilon_{1,2,2}^{(2)} \\ \frac{E\left[\lambda D_{2}+(\rho-\mu) D_{1}\right]}{(\rho+\lambda-\mu)(\rho-\mu)}-\frac{\lambda I_{2}}{\rho+\lambda}+B_{1,1}^{(2)} E^{\delta_{2}}-I_{1} & , E \geq \epsilon_{1,2,2}^{(2)}\end{cases}
$$

where $A_{1,1}^{(2)}<0$ and $B_{1,1}^{(2)}>0$ are determined via the value-matching and smooth-pasting conditions between the two branches of (13) and are indicated in (B-2) and (B-3) respectively. The first term in the top part of (13) is the expected value from operating the first version of the technology, while the second term reflects the option to invest in the second version, which is not available yet, and, therefore, must be adjusted via the third term. The first term in the bottom part of (13) reflects the expected present value of the revenues from the second version and the second term is the expected investment cost. Finally, the third term is the probability that the price will drop into the waiting region before the occurrence of the innovation. As in (9), a higher $\lambda$ increases the likelihood of the second innovation and reduces the relative loss in $\Phi_{1,1}^{(2)}(E)$, which, in turn, implies that $\Phi_{1,1}^{(2)}(E) \in\left[\Phi_{1,1}^{(1)}(E), F_{1,2,2}^{(2)}(E)\right) \forall \lambda \in \mathbb{R}^{+}$, as shown in Proposition 1. Note that a compulsive strategy implies investment in each version that becomes available, and, therefore, $m=\ell+1$ and $n=m+1$ $\forall \ell, m, n \in \mathbb{N}$.

Proposition $1 \forall \ell, m, n \in \mathbb{N}$ and $\forall \lambda \in \mathbb{R}^{+}, \Phi_{m, m}^{(n)}(E) \in\left[\Phi_{m, m}^{(m)}(E), F_{m, n, n}^{(n)}(E)\right)$.

Next, we step back and consider the option to invest in the first version that includes an embedded option to perform a single replacement. Notice that the value of an active project with a single embedded replacement option is described in (13) for $E<\epsilon_{1,2,2}^{(2)}$. Consequently, $F_{0,1,1}^{(2)}(E)$ is described in (14), where the top part reflects the value of the option to invest and the bottom part is the expected NPV at investment. Notice that the latter consists of the value from investment in the first version and a single embedded option to upgrade it when an innovation occurs.

$$
F_{0,1,1}^{(2)}(E)= \begin{cases}A_{0,1,1}^{(2)} E^{\beta_{1}} & , E<\epsilon_{0,1,1}^{(2)} \\ \Phi_{1,1}^{(2)}(E) & , E \geq \epsilon_{0,1,1}^{(2)}\end{cases}
$$


Although the optimal investment threshold, $\epsilon_{0,1,1}^{(2)}$, and the endogenous constant, $A_{0,1,1}^{(2)}$, are now obtained numerically via the value-matching and smooth-pasting conditions $(\mathrm{B}-4)$ and $(\mathrm{B}-5)$, we can investigate the impact of $\lambda$ on $\epsilon_{0,1,1}^{(2)}$ by expressing $F_{0,1,1}^{(2)}(E)$ as in (15).

$$
F_{0,1,1}^{(2)}(E)=\left(\frac{E}{\epsilon_{0,1,1}^{(2)}}\right)^{\beta_{1}}\left[\frac{D_{1} \epsilon_{0,1,1}^{(2)}}{\rho-\mu}-I_{1}+A_{1,2,2}^{(2)} \epsilon_{0,1,1}^{(2)^{\beta}}+A_{1,1}^{(2)} \epsilon_{0,1,1}^{(2)^{\delta_{1}}}\right], \forall E<\epsilon_{0,1,1}^{(2)}
$$

Then, the optimal investment rule is obtained by taking the first-order necessary condition (FONC) of (15) and is indicated in (16) where we equate the marginal benefit (MB) of delaying investment to the marginal cost (MC). By expressing the investment rule in this way, we can obtain further insights on the impact of technological uncertainty on the optimal investment threshold.

$$
\left(\frac{E}{\epsilon_{0,1,1}^{(2)}}\right)^{\beta_{1}}\left[\frac{D_{1}}{\rho-\mu}+\frac{\beta_{1}}{\epsilon_{0,1,1}^{(2)}} I_{1}-\beta_{1} A_{1,1}^{(2)} \epsilon_{0,1,1}^{(2)^{\delta_{1}-1}}\right]=\left(\frac{E}{\epsilon_{0,1,1}^{(2)}}\right)^{\beta_{1}}\left[\frac{\beta_{1} D_{1}}{\rho-\mu}-\delta_{1} A_{1,1}^{(2)} \epsilon_{0,1,1}^{(2)^{\delta_{1}-1}}\right]
$$

The first term on the left-hand side of (16) consists of the discount factor multiplied by the incremental project value created by waiting until the price is higher. This term is a positive, decreasing function of the output price, as waiting longer allows the project to start at a higher initial price, yet the rate at which this benefit accrues diminishes due to the effect of discounting. The second term represents the reduction in the MC of waiting due to saved investment cost. Similarly, the first term on the right-hand side reflects the opportunity cost of forgone cash flows discounted appropriately. Since $A_{1,1}^{(2)}<0$, the third and second terms on the left- and right-hand side, respectively, reflect the loss in option value from not having the second version yet. Specifically, the third term on the left-hand side is the MB from postponing the loss in value, whereas the second term on the right-hand side is the MC from a potentially greater impact of the loss from waiting for a higher threshold price. Notice that it is the impact of $\lambda$ on these two terms that determines the overall behaviour of the $\epsilon_{0,1,1}^{(2)}$ with respect to $\lambda$, and, as Proposition 2 indicates more generally, the impact of $\lambda$ on $\epsilon_{\ell, m, m}^{(m)}$ is non-monotonic. Intuitively, a low $\lambda$ facilitates a compulsive strategy by allowing for enough time between the arrival of subsequent innovations, yet a high $\lambda$ lowers the feasibility of this strategy, thereby motivating earlier technology adoption.

Proposition 2 Under a compulsive strategy, $\forall \ell, m, n \in \mathbb{N}$ the impact of $\lambda$ on $\epsilon_{\ell, m, m}^{(n)}$ is non-monotonic.

Finally, we step back to state $(0,0)$ in order to determine the value of a project with two embedded replacement options that are subject to the arrival of the corresponding technologies. Unlike (7), now the value of the first investment option, $F_{0,1,1}^{(2)}(E)$, includes a single embedded option to perform one upgrade and that, as long as $\epsilon_{0,1,1}^{(2)}<\epsilon_{1,2,2}^{(2)}$, the solution depends on whether $E<\epsilon_{0,1,1}^{(2)}$ or $E \geq \epsilon_{0,1,1}^{(2)}$. For $E<\epsilon_{0,1,1}^{(2)}$, it would still be optimal to delay investment even if the first version became available, whereas for $E \geq \epsilon_{0,1,1}^{(2)}$, investment should occur immediately. The expression for $\Phi_{0,0}^{(2)}(E)$ is indicated in $(17)$, where $A_{0,0}^{(2)}<0$ and $B_{0,0}^{(2)}>0$ are obtained via the value-matching and smooth-pasting conditions between the two branches and are indicated in (B-7) and 
(B-8) respectively.

$$
\Phi_{0,0}^{(2)}(E)= \begin{cases}A_{0,1,1}^{(2)} E^{\beta_{1}}+A_{0,0}^{(2)} E^{\delta_{1}} & , E<\epsilon_{0,1,1}^{(2)} \\ \frac{\lambda D_{1} E}{(\rho+\lambda-\mu)(\rho-\mu)}-\frac{\lambda I_{1}}{\rho+\lambda}+A_{1,2,2}^{(2)} E^{\beta_{1}}+A_{1,1}^{(2)} E^{\delta_{1}}+B_{0,0}^{(2)} E^{\delta_{2}} & , E \geq \epsilon_{0,1,1}^{(2)}\end{cases}
$$

Likewise, we can determine the optimal investment threshold and the value of the option to invest for a project with any number of replacement options under a compulsive strategy.

\section{Leapfrog versus Laggard Strategy}

It is possible that a better version of the technology becomes available while a firm waits in order to invest in an existing one, thus replacing the initial investment option with the option to choose between two alternatives. Here, we assume that a firm would not want to adopt an existing version before comparing it to the next one. Consequently, the transition from $(0,1,1)$ to $(1,1)$ is not possible, and the only state prior to $(1,2,2)$ is $(0,1 \vee 2)$, from which the firm may either adopt a laggard strategy and invest in the first version with the embedded option to upgrade to the second or $(\mathrm{V})$ adopt a leapfrog strategy and invest directly in the second version (Figure 3). Proposition 3 indicates the condition that is required in order to have a tradeoff between the two subsequent technology versions.

Proposition 3 A tradeoff between two technology versions $\ell$ and $n$ exists if $\frac{D_{n}}{\sum_{i=1}^{n} I_{i}}<\frac{D_{\ell}}{\sum_{i=1}^{\ell} I_{i}}, \forall \ell, n$ with $\ell<n$.

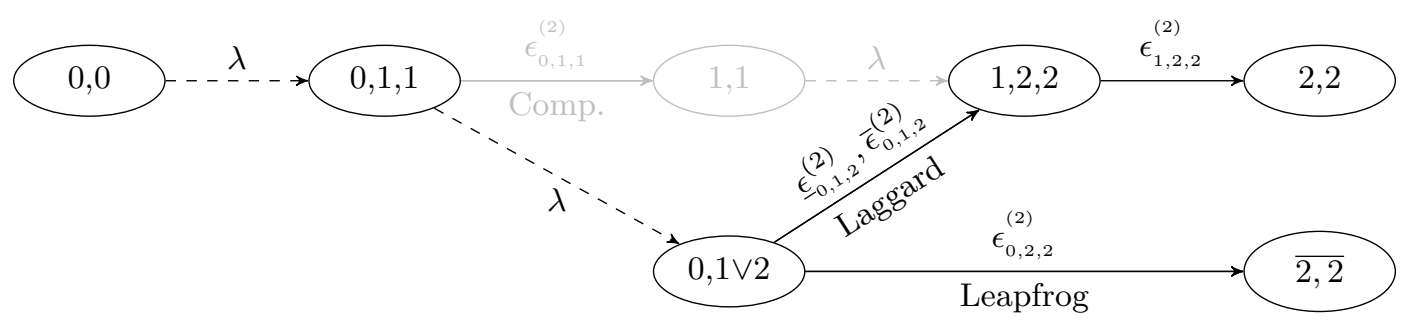

Fig. 3 State transition diagram for $N=2$ under leapfrog and laggard strategy

Since the analysis related to states $(2,2)$ and $(1,2,2)$ is the same as in Section 5 , we proceed directly to state $(\overline{2,2})$. Here, if the firm adopts the second version directly from $(0,1 \vee 2)$, then it does not incur the cost $I_{1}$, and the expected NPV from immediate investment is indicated in (18).

$$
\Phi_{\overline{2,2}}^{(2)}(E)=\frac{D_{2} E}{\rho-\mu}-I_{2}
$$

Next, we consider state $(0,1 \vee 2)$ where the firm has the option to choose either the first version of the technology with the option to switch to the second or the second version directly. Due to the presence of the second version, there exist two waiting regions, i.e., $\left(0, \underline{\epsilon}_{0,1,2}^{(2)}\right]$ and $\left[\bar{\epsilon}_{0,1,2}^{(2)}, \epsilon_{0,2,2}^{(2)}\right]$. If $E<\underline{\epsilon}_{0,1,2}^{(2)}$, then the firm will adopt a laggard strategy, i.e., wait until $E=\underline{\epsilon}_{0,1,2}^{(2)}$ and invest in the first version. Intuitively, the output price is too low to 
justify investment in the more expensive technology version, and, therefore, investment in only the relatively cheaper option is feasible. Alternatively, if $E \in\left[\bar{\epsilon}_{0,1,2}^{(2)}, \epsilon_{0,2,2}^{(2)}\right]$, then the firm can either adopt a laggard or a leapfrog strategy. Specifically, if the output price increases to $\epsilon_{0,2,2}^{(2)}$, then the firm will invest in the second version, but if it drops to $\bar{\epsilon}_{0,1,2}^{(2)}$, then it will invest in the first one. Consequently, the laggard strategy is adopted either when the output price is low, i.e., $E<\underline{\epsilon}_{0,1,2}^{(2)}$, and increases to $\underline{\epsilon}_{0,1,2}^{(2)}$ or when the output price is high, i.e., $E \in\left[\bar{\epsilon}_{0,1,2}^{(2)}, \epsilon_{0,2,2}^{(2)}\right]$, and decreases to $\bar{\epsilon}_{0,1,2}^{(2)}$.

As a result, assuming that $\underline{\epsilon}_{0,1,2}^{(2)}<\epsilon_{1,2,2}^{(2)}$, according to Décamps et al. (2006), the value function in state $(0,1 \vee 2)$ is indicated in (19), where $A_{0,1 \vee 2}^{(2)}$ and $\underline{\epsilon}_{0,1,2}^{(2)}$ are determined via the value-matching and smooth-pasting conditions between the first two branches, while $\bar{\epsilon}_{0,1,2}^{(2)}, \epsilon_{0,2,2}^{(2)}, G_{0,1 \vee 2}^{(2)}$, and $H_{0,1 \vee 2}^{(2)}$ are obtained numerically via the second, third, and fourth branch.

$$
F_{0,1 \vee 2}^{(2)}(E)= \begin{cases}A_{0,1 \vee 2}^{(2)} E^{\beta_{1}} & , E<\underline{\epsilon}_{0,1,2}^{(2)} \\ F_{1,2,2}^{(2)}(E) & , \underline{\epsilon}_{0,1,2}^{(2)} \leq E \leq \bar{\epsilon}_{0,1,2}^{(2)} \\ G_{0,1 \vee 2}^{(2)} E^{\beta_{2}}+H_{0,1 \vee 2}^{(2)} E^{\beta_{1}} & , \bar{\epsilon}_{0,1,2}^{(2)}<E<\epsilon_{0,2,2}^{(2)} \\ \Phi_{\overline{2,2}}^{(2)}(E) & , E \geq \epsilon_{0,2,2}^{(2)}\end{cases}
$$

Interestingly, although now both versions are available and, as a result, there is no loss in the value of the option to invest in the first one, the corresponding investment threshold under a laggard strategy when $E<\underline{\epsilon}_{0,1,2}^{(2)}$ is greater than that under a compulsive strategy when the arrival of the second innovation is uncertain, i.e., $\underline{\epsilon}_{0,1,2}^{(2)}>\epsilon_{0,1,1}^{(2)}$, whereas $\underline{\epsilon}_{0,1,2}^{(2)}=\epsilon_{0,1,1}^{(2)}$ when $\lambda=0$ or $\lambda \rightarrow \infty$. More generally, Proposition 4 shows that investment in the first version is not affected by the absence $(\lambda=0)$ or presence $(\lambda \rightarrow \infty)$ of the second one and that technological uncertainty may actually accelerate investment, i.e., it increases the incentive to adopt the current version when the arrival of a subsequent one is uncertain.

Proposition 4 Under a leapfrog or laggard strategy, $\forall \ell, m, n \in \mathbb{N}$ we have $\epsilon_{\ell, m, m}^{(n)}<\underline{\epsilon}_{\ell, m, n}^{(n)} \forall \lambda \in(0,+\infty)$, whereas $\lambda=0 \Rightarrow \epsilon_{\ell, m, m}^{(n)}=\underline{\epsilon}_{\ell, m, n}^{(n)}$ and $\lambda \rightarrow \infty \Rightarrow \epsilon_{\ell, m, m}^{(n)} \rightarrow \underline{\epsilon}_{\ell, m, n}^{(n)}$.

The value function in state $(0,1,1)$ is described in $(20)$. The first term on the right-hand side is the option to invest in the first version with an embedded option to upgrade to the second one if no innovation takes place within the time interval $d t$. However, as the second term indicates, if an innovation occurs during $d t$, then the firm obtains the option to choose between two versions.

$$
F_{0,1,1}^{(2)}(E)=(1-\rho d t)\left[(1-\lambda d t) \mathbb{E}_{E}\left[F_{0,1,1}^{(2)}(E+d E)\right]+\lambda d t \mathbb{E}_{E}\left[F_{0,1 \vee 2}^{(2)}(E+d E)\right]\right]
$$

Notice that (20) has to be solved separately for each of the four regions of $E$ that are indicated in (19). By substituting for $F_{0,1 \vee 2}^{(2)}(E)$ in $(20)$ and solving for $F_{0,1,1}^{(2)}(E)$, we obtain the solution indicated in $(21)$, where $A_{1,2,2}^{(2)}$ is described in (12) and the endogenous constants $A_{0,1,1}^{(2)}, L_{0,1,1}^{(2)}, P_{0,1,1}^{(2)}, Q_{0,1,1}^{(2)}, R_{0,1,1}^{(2)}$, and $J_{0,1,1}^{(2)}$ are determined 
via the value-matching and smooth-pasting conditions between the branches of (21).

$$
F_{0,1,1}^{(2)}(E)= \begin{cases}A_{0,1 \vee 2}^{(2)} E^{\beta_{1}}+P_{0,1,1}^{(2)} E^{\delta_{1}} & , E<\underline{\epsilon}_{0,1,2}^{(2)} \\ \frac{\lambda D_{1} E}{(\rho+\lambda-\mu)(\rho-\mu)}-\frac{\lambda I_{1}}{\rho+\lambda}+A_{1,2,2}^{(2)} E^{\beta_{1}}+L_{0,1,1}^{(2)} E^{\delta_{1}}+B_{0,1,1}^{(2)} E^{\delta_{2}} & , \underline{\epsilon}_{0,1,2}^{(2)} \leq E \leq \bar{\epsilon}_{0,1,2}^{(2)} \\ G_{0,1 \vee 2}^{(2)} E^{\beta_{2}}+H_{0,1 \vee 2}^{(2)} E^{\beta_{1}}+Q_{0,1,1}^{(2)} E^{\delta_{1}}+R_{0,1,1}^{(2)} E^{\delta_{2}} & , \bar{\epsilon}_{0,1,2}^{(2)}<E<\epsilon_{0,2,2}^{(2)} \\ \frac{\lambda D_{2} E}{(\rho+\lambda-\mu)(\rho-\mu)}-\frac{\lambda I_{2}}{\rho+\lambda}+J_{0,1,1}^{(2)} E^{\delta_{2}} & , E \geq \epsilon_{0,2,2}^{(2)}\end{cases}
$$

Finally, like in Section 5, we can determine the value function in state $(0,0)$. Without loss of generality, the comparison of $F_{0,1,1}^{(2)}(E)$ under the two strategies can be done in state $(0,1,1)$, and, therefore, the analysis of state $(0,0)$ is omitted. In fact, since we know from Proposition 4 that $\epsilon_{0,1,1}^{(2)} \leq \underline{\epsilon}_{0,1,2}^{(2)}$, the comparison of the strategies at $(0,1,1)$ can be made separately for each of the regions of $E$ that are indicated in (14) and (21).

\section{Comparison of the Strategies}

Despite the incentive to delay investment in an old version of the technology in order to compare it with a newer one, it is the interaction between price and technological uncertainty that determines the optimal strategy. Here, we extend Section 6 by assuming that the choice of strategy depends on $E$ and $\lambda$, and, thus, it is not determined exogenously. Hence, both $(0,1,1) \rightarrow(1,1)$ and $(0,1,1) \rightarrow(0,1 \vee 2)$ are possible transitions, and the final choice of strategy is endogenous. Figure 4 summarises the possible strategies for different values of $E$ and $\lambda$ from state $(0,1,1)$. As shown in Proposition 5 , the compulsive strategy is optimal $\forall \lambda \in \mathbb{R}^{+}$within the first two price regions, i.e., $E \leq \bar{\epsilon}_{0,1,2}^{(2)}$. Intuitively, even if a second version of the technology were available, then a firm would have to wait long before the output price reaches the corresponding investment threshold, and the expected payoff from investment in the second version does not offset the forgone revenues from skipping the first one. By contrast, in the third and fourth regions, i.e., $E>\bar{\epsilon}_{0,1,2}^{(2)}$, the optimal strategy depends on $\lambda$. Indeed, the required $\lambda$ for which a firm may consider waiting for the next version decreases as the output price increases, and, as shown in Proposition 6, it is possible to determine the required value of $\lambda \forall E>\bar{\epsilon}_{0,1,2}^{(2)}$. Additionally, high price uncertainty delays investment in both the first and the second version and decreases the feasibility of a laggard strategy in state $(0,1 \vee 2)$ by increasing $\underline{\epsilon}_{0,1,2}^{(2)}$ and lowering $\bar{\epsilon}_{0,1,2}^{(2)}$, thereby increasing the likelihood of adopting a leapfrog strategy.

More specifically, notice that in the first two regions, i.e., $E \in\left(0, \bar{\epsilon}_{0,1,2}^{(2)}\right]$, the compulsive strategy always dominates the leapfrog/laggard strategy (Proposition 5). Indeed, a leapfrog/laggard strategy forces the firm to wait for the second version, and, therefore, the expected payoff is conditional on its arrival. Hence, $F_{0,1,1}^{(2)}(E)$ is greater under a compulsive strategy $\forall \lambda \in \mathbb{R}^{+}$since the firm receives the same payoff without having to wait for the second version. Consequently, if $E \in\left(0, \bar{\epsilon}_{0,1,2}^{(2)}\right]$, then the firm does not need to wait until $\bar{\epsilon}_{0,1,2}^{(2)}$ in order to invest in the first version (Décamps et al., 2006), since, under a compulsive strategy, the investment option should be exercised at $\epsilon_{0,1,1}^{(2)} \leq \underline{\epsilon}_{0,1,2}^{(2)}$. 


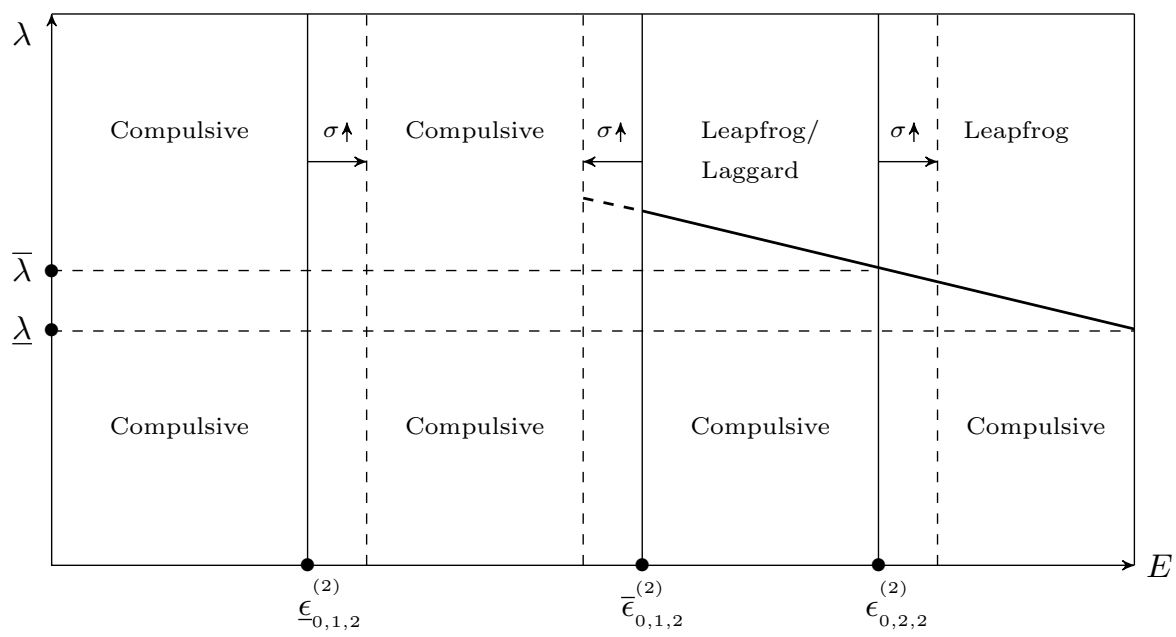

Fig. 4 Comparison of the strategies in $(0,1,1)$

Proposition $5 \forall E \in\left(0, \bar{\epsilon}_{0,1,2}^{(2)}\right]$, if the investment region in state $(0,1 \vee 2)$ is dichotomous, then the compulsive strategy dominates the leapfrog/laggard strategy $\forall \lambda \in \mathbb{R}^{+}$.

By contrast, when the output price is high, i.e., $E>\bar{\epsilon}_{0,1,2}^{(2)}$, it is possible that a laggard or a leapfrog strategy dominates. Intuitively, a high output price compensates for a low $\lambda$ and increases the incentive to wait for the second version of the technology, while a low output price increases the rate $\lambda$ for which a firm may consider waiting for the second version. More specifically, if $E \in\left(\bar{\epsilon}_{0,1,2}^{(2)}, \epsilon_{0,2,2}^{(2)}\right]$, then the payoff from a compulsive strategy must be compared to the value of the option to choose either one of the two versions. In effect, the value of the option to choose between two versions may be higher than the expected NPV from adopting the older one if the rate of innovation in high. Similarly, if $E \in\left(\epsilon_{0,2,2}^{(2)}, \infty\right)$, then the value from immediate investment in the second version, which under a leapfrog strategy is contingent upon its arrival, must be compared to the value from immediate investment in the first version with an embedded option to upgrade it. If $\lambda$ is high, then it may be preferable to wait for the second version rather than invest in the first one. As Proposition 6 indicates, $\forall E \in\left(\bar{\epsilon}_{0,1,2}^{(2)}, \infty\right]$ it is possible to determine the optimal strategy for each pair $(E, \lambda)$ as well as the minimum rates $\underline{\lambda}$ and $\bar{\lambda}$ for which a firm would adopt a leapfrog or a leapfrog/laggard strategy respectively.

Proposition $6 \exists \underline{\lambda}, \bar{\lambda} \in \mathbb{R}^{+}$with $\underline{\lambda} \leq \bar{\lambda}$ :

$\mathrm{i}: \forall \lambda>\underline{\lambda}, \exists \mathcal{B} \subseteq\left(\epsilon_{0,2,2}^{(2)}, \infty\right): \forall E \in \mathcal{B}$, the leapfrog strategy dominates

ii : $\forall \lambda>\bar{\lambda}, \exists \mathcal{A} \subset\left(\bar{\epsilon}_{0,1,2}^{(2)}, \epsilon_{0,2,2}^{(2)}\right]: \forall E \in \mathcal{A}$, the leapfrog/laggard strategy dominates

Hence, by taking into account the arrival of innovations, we obtain the maximised value in state $(0,1,1)$, which is described in (22). The first two branches of (22) indicate that the compulsive strategy is always better for $E<\bar{\epsilon}_{0,1,2}^{(2)}$ regardless of $\lambda$. However, as the bottom two branches indicate, for $E>\bar{\epsilon}_{0,1,2}^{(2)}$ the optimal strategy 
depends on $E$ and $\lambda$.

$$
F_{0,1,1}^{(2)}(E)=\left\{\begin{array}{cl}
A_{0,1,1}^{(2)} E^{\beta_{1}} & , E<\underline{\epsilon}_{0,1,2}^{(2)} \\
\Phi_{1,1}^{(2)}(E)+A_{1,2,2}^{(2)} E^{\beta_{1}}+A_{1,1}^{(2)} E^{\delta_{1}} & , \underline{\epsilon}_{0,1,2}^{(2)}<E<\bar{\epsilon}_{0,1,2}^{(2)} \\
\max _{E, \lambda}\left\{\Phi_{1,1}^{(2)}(E)+A_{1,2,2}^{(2)} E^{\beta_{1}}+A_{1,1}^{(2)} E^{\delta_{1}},\right. & \\
\left.G_{0,2}^{(2)} E^{\beta_{2}}+H_{0,2}^{(2)} E^{\beta_{1}}+Q_{0,1,1}^{(2)} E^{\delta_{1}}+R_{0,1,1}^{(2)} E^{\delta_{2}}\right\} & , \bar{\epsilon}_{0,1,2}^{(2)}<E<\epsilon_{0,2,2}^{(2)} \\
\max _{E, \lambda}\left\{\Phi_{1,1}^{(2)}(E)+A_{1,2,2}^{(2)} E^{\beta_{1}}+A_{1,1}^{(2)} E^{\delta_{1}},\right. & \\
\left.\frac{\lambda D_{2} E}{(\rho+\lambda-\mu)(\rho-\mu)}-\frac{\lambda I_{2}}{\rho+\lambda}+J_{0,1,1}^{(2)} E^{\delta_{2}}\right\} & , E \geq \epsilon_{0,2,2}^{(2)}
\end{array}\right.
$$

\section{Numerical Examples}

\subsection{Compulsive Strategy with $\mathrm{N}=1$}

For the numerical examples, the values of the different parameters are $\rho=0.1, \mu=0.01$, and $\sigma \in[0,0.3]$. Also, for the purposes of the analysis, we assume that $\lambda \in[0,1]$. We begin with the case of $N=1$ and set $I_{1}=1500$ and $D_{1}=16$. The left panel of Figure 5 illustrates the value of a single investment option, $F_{0,1,1}^{(1)}(\cdot)$, the expected NPV from exercising it, $\Phi_{1,1}^{(1)}(\cdot)$, and the expected NPV from performing a single upgrade that is not available yet, $\Phi_{0,0}^{(1)}(\cdot)$. Notice that the value function in state $(0,0)$ increases with higher $\lambda$. Indeed, a higher $\lambda$ increases the likelihood of an innovation, thereby raising the value function $\Phi_{0,0}^{(1)}(\cdot)$. Once a new version of the technology arrives, the firm has the option to adopt it, and the optimal investment threshold is $\epsilon_{0,1,1}^{(1)}=14.06$. Notice that there are no additional investment options available to influence the value of the initial investment decision. As a result, conditional on the arrival of an innovation, the likelihood of exercising the investment option in state $(0,1,1)$ is subject only to the underlying stochastic process. Consequently, as indicated in (5), $\lambda$ impacts the likelihood of an innovation but not the optimal investment threshold.

\subsection{Compulsive Strategy with $\mathrm{N}=2$}

Next, we extend the case of compulsive strategy by assuming that a firm holds two investment options. The right panel of Figure 5 illustrates the value functions in states $(2,2),(1,2,2),(1,1),(0,1,1)$, and $(0,0)$ for $\lambda=0.01$. The numerical assumptions $I_{1}=500, D_{1}=8, I_{2}=1500$, and $D_{2}=16$, imply that the second version is three times as expensive as the first one and twice as efficient, i.e., they satisfy the assumption $\frac{D_{2}}{I_{1}+I_{2}}<\frac{D_{1}}{I_{1}}$. Like in the case $N=1$, the value function $F_{1,2,2}^{(2)}(\cdot)$ and the investment threshold $\epsilon_{1,2,2}^{(2)}$ are independent of $\lambda$. For $\lambda=0.01$, the value function in state $(0,0)$ increases with $E$ since now both of the embedded investment options have a positive value. Also, the value function $F_{0,1,1}^{(2)}(\cdot)$ is greater than $\Phi_{0,0}^{(2)}(\cdot)$ since in state $(0,0)$ the availability of both versions is uncertain, while, at the same time, it is lower than $F_{1,2,2}^{(2)}(\cdot)$ where both versions are available, as shown in Proposition 1. 
Fig. 5 Option and project value under a compulsive strategy with $N=1$ (left) and $N=2$ with $\lambda=0.01$ (right) for $\sigma=0.2$

Fig. 6 Relative loss in option value (left) and optimal investment threshold (right) versus $\sigma, \lambda$

The left panel in Figure 6 illustrates the relative loss in the value function, $\Phi_{1,1}^{(2)}(\cdot)$, due to the likelihood associated with the arrival of the second version. This is expressed in $(23)$, where $F_{1,2,2}^{(2)}(E)$ is the value of the option to invest when the first version is in operation and the second version is available for adoption.

$$
\frac{F_{1,2,2}^{(2)}(E)-\Phi_{1,1}^{(2)}(E)}{F_{1,2,2}^{(2)}(E)}, E \leq \epsilon_{1,2,2}^{(2)}
$$

Notice that for $\lambda=0$, we have $A_{1,1}^{(2)}=-A_{1,2,2}^{(2)}$, which implies that $\Phi_{1,1}^{(2)}(\cdot)=\Phi_{1,1}^{(1)}(\cdot)$. Thus, for $\lambda=0$ the relative loss in $\Phi_{1,1}^{(2)}(\cdot)$ is maximised since the value of the embedded option to invest in the second version is zero. Then, for low values of $\lambda$, the relative loss in $\Phi_{1,1}^{(2)}(\cdot)$ decreases quickly because a higher $\lambda$ increases the likelihood of the arrival of the second version, thus decreasing the loss in option value due to technological uncertainty. Above a certain value of $\lambda$, the decrease is less pronounced since the likelihood of at least one innovation occurring converges to one. Thus, as $\lambda$ increases, $\Phi_{1,1}^{(2)}(\cdot) \rightarrow F_{1,2,2}^{(2)}(E)$, which implies that the relative loss in option value converges to zero, as shown in Proposition 1. As the right panel illustrates, for low values of $\lambda$ the rapid decrease in the relative loss in $\Phi_{1,1}^{(2)}(\cdot)$ increases the incentive to invest and lowers the required investment threshold, $\epsilon_{0,1,1}^{(2)}$. Surprisingly, however, $\epsilon_{0,1,1}^{(2)}$ does not always decrease with higher $\lambda$. Indeed, when $\lambda$ is low, $\epsilon_{0,1,1}^{(2)}$ decreases as the rate of innovation increases; however, as $\lambda$ increases further, $\epsilon_{0,1,1}^{(2)}$ increases and converges to its value for $\lambda=0$, as shown in Proposition 4. Intuitively, when the rate of innovation is low, the extra benefit due to the decrease in the relative loss in $\Phi_{1,1}^{(2)}(\cdot)$ is more pronounced than the incentive to delay investment. By contrast, when innovations arrive more frequently, the decrease in the relative loss in $\Phi_{1,1}^{(2)}(\cdot)$ is less pronounced than the value of waiting, which increases with higher uncertainty. Finally, uncertainty raises the value of the investment opportunity, thereby increasing the incentive to delay investment, and, in turn, the optimal investment threshold, while, at the same time, it makes the non-monotonic impact of $\lambda$ on $\epsilon_{0,1,1}^{(2)}$ more pronounced.

Figure 7 illustrates the $\mathrm{MB}$ and $\mathrm{MC}$ of delaying investment for $\sigma=0.2$ and $\lambda=0,0.1,0.3$. Notice that, as $\lambda$ increases, both the MB and the MC of delaying investment decrease because the frequent arrival of new versions erodes the value of waiting to invest in the currently available version and lowers the forgone revenues from waiting. Intuitively, for low $\lambda$, versions are not arriving frequently, and the time interval between subsequent versions may be large enough to allow the adoption of a version before the next one becomes available. However, a higher $\lambda$ decreases the time interval between subsequent versions, and, in the attempt to maintain a compulsive strategy, a firm would be more willing to adopt the current version sooner before the unreleased version becomes available, as shown in Proposition 4. In line with the right panel of Figure 6, higher $\lambda$ implies that innovations take place more frequently, and, therefore, the incentive to delay investment in order to avoid making a mistake increases. Thus, the impact of a higher $\lambda$ on the optimal investment threshold under 
Fig. $7 \mathrm{MB}$ and $\mathrm{MC}$ of delaying investment for, $\sigma=0.2$ and $\lambda=0,0.1,0.3$

Fig. 8 Option and project value for $N=2, \sigma=0.2$ under leapfrog and laggard strategy (left) and impact of $\sigma$ on $\underline{\epsilon}_{0,1,2}^{(2)}, \bar{\epsilon}_{0,1,2}^{(2)}, \epsilon_{0,2,2}^{(2)}$, and $\epsilon_{1,2,2}^{(2)}$ for $\lambda=0.1$

a compulsive strategy reflects the limitations of this strategy, and, in turn, the point at which a transition to a leapfrog/laggard strategy may be necessary.

\subsection{Leapfrog versus Laggard Strategy}

The left panel in Figure 8 illustrates the value functions in the states $(0,1 \vee 2)$ and $(0,1,1)$, when the transition from $(0,1,1)$ to $(1,1)$ is not considered. If $E<9.37$, then the firm must wait until $E=9.37$ and adopt the first version of the technology. Also, if $E \in[10.96,14.51]$, then, due to the presence of the second version, the firm has to wait again, i.e., refrain from adopting any versions. Specifically, if $E$ increases to 14.51 , then the firm will invest directly in the second version, but if $E$ drops to 10.96, then it will invest in the first version while holding the option to switch to the second. Notice that the value function $F_{0,1,1}^{(2)}(\cdot)$ is lower than $F_{0,1 \vee 2}^{(2)}(\cdot)$ because $F_{0,1 \vee 2}^{(2)}(\cdot)$ reflects the value of the option to choose between two versions that are available, whereas $F_{0,1,1}^{(2)}(\cdot)$ reflects the same value function in the absence of the second version. However, as $\lambda$ increases, the loss in value due to the likelihood associated with the arrival of the second version decreases and $F_{0,1,1}^{(2)}(\cdot)$ converges to $F_{0,1 \vee 2}^{(2)}(\cdot)$. The impact of volatility on the optimal investment thresholds $\underline{\epsilon}_{0,1,2}^{(2)}, \bar{\epsilon}_{0,1,2}^{(2)}, \epsilon_{1,2,2}^{(2)}$, and $\epsilon_{0,2,2}^{(2)}$ is illustrated in the right panel. Notice that, since the investment thresholds $\underline{\epsilon}_{0,1,2}^{(2)}$ and $\bar{\epsilon}_{0,1,2}^{(2)}$ move in opposite directions with higher volatility, it is possible that $\underline{\epsilon}_{0,1,2}^{(2)}>\bar{\epsilon}_{0,1,2}^{(2)}$. In that case, the region of direct investment in the first version disappears, and, therefore, it is optimal to adopt a leapfrog strategy, i.e., wait until $E=\epsilon_{0,2,2}^{(2)}$ and then invest in the second version. Intuitively, price uncertainty increases both waiting regions, and, consequently, beyond a certain, high level of uncertainty these regions will overlap, thereby forming a single waiting region. Notice also that, although $\lambda$ raises the value function in state $(0,1,1)$, it does not impact the investment thresholds in both the left and the right panels. This happens because these thresholds are determined under the assumption of a leapfrog/laggard strategy, whereby the firm waits for both technology versions to become available before deciding which one to invest in, and, therefore, there is no technological uncertainty.

\subsection{Comparison of the Strategies}

In order to compare the three strategies, we assume that $\sigma \leq 0.28$, since, according to the right panel of Figure 8 , for $\sigma>0.28$ the laggard strategy is not feasible in state $(0,1 \vee 2)$. The left panel in Figure 9 illustrates the relative value of the compulsive and the leapfrog/laggard strategies for $E<\underline{\epsilon}_{0,1,2}^{(2)}$. More specifically, it compares the first branch of (14) with the first branch of (21) as in (24).

$$
\frac{A_{0,1,1}^{(2)} E^{\beta_{1}}}{A_{0,1 \vee 2}^{(2)} E^{\beta_{1}}+P_{0,1,1}^{(2)} E^{\delta_{1}}}
$$


Fig. 9 Relative value of the two strategies under low, i.e., $E<\underline{\epsilon}_{0,1,2}^{(2)}$, (left) and high output price, i.e., $\bar{\epsilon}_{0,1,2}^{(2)}<E<\epsilon_{0,2,2}^{(2)}$ (thick lines) and $E>\epsilon_{0,2,2}^{(2)}>\epsilon_{0,1,1}^{(2)}$ (thin lines), (right)

Similarly, the right panel compares the two strategies for $\bar{\epsilon}_{0,1,2}^{(2)}<E<\epsilon_{0,2,2}^{(2)}$ (thick lines) and $E>\epsilon_{0,2,2}^{(2)}>\epsilon_{0,1,1}^{(2)}$ (thin lines), i.e., the second branch of (14) with the third and fourth branch of (21), as indicated in (25).

$$
\frac{\Phi_{1,1}^{(1)}(E)+A_{1,2,2}^{(2)} E^{\beta_{1}}+A_{1,1}^{(2)} E^{\delta_{1}}}{G_{0,1 \vee 2}^{(2)} E^{\beta_{2}}+H_{0,1 \vee 2}^{(2)} E^{\beta_{1}}+Q_{0,1,1}^{(2)} E^{\delta_{1}}+R_{0,1,1}^{(2)} E^{\delta_{2}}} \text { and } \frac{\Phi_{1,1}^{(1)}(E)+A_{1,2,2}^{(2)} E^{\beta_{1}}+A_{1,1}^{(2)} E^{\delta_{1}}}{\frac{\lambda D_{2} E}{(\rho+\lambda-\mu)(\rho-\mu)}-\frac{\lambda I_{2}}{\rho+\lambda}+J_{0,1,1}^{(2)} E^{\delta_{2}}}
$$

According to the left panel, it is always better to adopt a compulsive over a leapfrog/laggard strategy when the output price is low because, even if a second version were available, then it would still be optimal to delay investment until $E=\underline{\epsilon}_{0,1,2}^{(2)}$ and subsequently invest in the first version. Notice also that the relative value of the compulsive strategy increases as output price uncertainty decreases. This happens because a more stable economic environment reduces the likelihood of an unexpected increase in the output price, and, in turn, the opportunity cost from adopting the strategy. By contrast, as the right panel illustrates, the relative value of the compulsive and leapfrog/laggard strategies can drop below one when $\lambda$ and $E$ are high, thereby indicating that the expected value from adopting a leapfrog or a laggard strategy exceeds that of the compulsive strategy. This happens because a higher output price reduces the expected time until investment in the second version is justified, while, at the same time, a high $\lambda$ decreases the feasibility of the compulsive strategy by reducing the expected time between subsequent innovations. Again, this result is more pronounced under lower price uncertainty since this decreases the likelihood of an unexpected downturn. Finally, in line with Figure 4, a higher output price compensates for technological uncertainty by reducing the required rate of innovation for which a firm is willing to ignore an available version and wait for a new one. For example, under high price uncertainty and low output price, a firm would require a rate $\bar{\lambda}=0.7$ in order to consider waiting for a new version instead of investing in the current one, whereas a higher output price lowers the required rate of innovation to $\underline{\lambda}=0.62$.

While these results are in line with Grenadier and Weiss (1997), we extend their framework by modelling price uncertainty via a continuous-time stochastic process in addition to a representation of technological uncertainty. This not only enables the derivation of price thresholds corresponding to each strategy but also facilitates the analysis of the endogenous relation between price and technological uncertainty and its impact on the optimal strategy selection decision. Indeed, while we confirm that the likelihood of adopting a laggard or a leapfrog strategy increases as the rate of innovation increases, we also show that higher price uncertainty may reduce the feasibility of a laggard strategy and lower the attractiveness of a leapfrog strategy, thus increasing the incentive to adopt a compulsive strategy. 


\section{Conclusions}

We develop a real options framework for sequential investment in order to address the problem of optimal replacement of an emerging technology that is subject to frequent upgrades, taking into account price and technological uncertainty. Although such features are crucial for investment in many industries, analytical formulations of sequential investment that include both of them are limited. Consequently, we extend the real options approach by incorporating these features into an analytical framework for sequential investment in order to obtain insights on their combined impact on the investors' propensity to upgrade equipment. The main contribution of this paper is that it illustrates how price and technological uncertainty interact to affect not only the optimal technology-adoption strategy but also the associated investment rule. This provides more detailed insights on the combined impact of these features on an investor's propensity to upgrade equipment than what the traditional literature has so far indicated, e.g., Grenadier and Weiss (1997).

We find that, under a compulsive strategy, an increase in the rate of innovation does not affect the optimal investment threshold when a firm holds a single investment option, even though it increases the likelihood of an innovation. Interestingly, under multiple investment opportunities, a higher rate of innovation increases the value of an investment opportunity, yet the corresponding investment threshold may either increase or decrease. In fact, uncertainty regarding the arrival of innovations may actually accelerate investment in a version. Moreover, a comparison of the strategies indicates that, when the output price is low, the compulsive strategy is always better as long as, under a leapfrog/laggard strategy, the investment region is dichotomous. However, when the rate of innovation is high, it is possible that the option to choose between two versions is more valuable than the immediate payoff from investment in the first version with an embedded option to switch to the second.

The implications of the results are crucial for the participants of many industries, e.g., RE, pharmaceutical, and telecommunications, who, in the light of increasing economic uncertainty face the problem of investing in products that may become obsolete. For example, in the RE sector, the rate of innovation may be high but, as indicated in the right panel of Figure 9, price uncertainty reduces the relative value of a leapfrog strategy, thereby making investors more reluctant in adopting new technology versions. Similarly, the overall success rate for drugs moving through clinical trials to FDA approval is generally low, and, in line with Figure 4, this raises the value of a compulsive relative to a leapfrog/laggard strategy. By contrast, a leapfrog/laggard strategy may be more pertinent to the smartphone industry, where the rate of innovation is high yet low price uncertainty enables consumers to wait for new versions. Hence, such a framework may allow investors in RE equipment to develop more informed adoption strategies and enable companies to reduce their exposure to technological obsolescence by anticipating any changes in the rate that technologies become available. Similarly, policy measures for supporting RE via R\&D funding can become more efficient by taking into account the investors' propensity to adopt new technologies. 
Apart from market and technological uncertainty it is possible to analyse policy uncertainty with respect to any change of a support scheme via a regime-switching model (Boomsma et al., 2012). Hence, the current framework can be extended to analyse not only the impact of policy uncertainty on investment and operational decisions but also the endogenous relation between technological and policy uncertainty. Additionally, it would be interesting to relax the assumption of risk neutrality and study how risk aversion due to technical risk affects the optimal investment policy via a utility-based framework (Chronopoulos et al., 2013), allow for strategic interactions between competing investors and developers, or a different stochastic process in order to relax the limitations inherent in the GBM. Finally, extensions in the same line of work may be motivated also by empirical research that studies the relationship between investment cost and the future development path of emerging technologies (MacGillivray et al., 2014).

\section{APPENDIX}

Compulsive Strategy with $\mathrm{N}=1$

The value of the investment option in state $(0,1,1)$ is indicated in $(\mathrm{A}-1)$.

$$
F_{0,1,1}^{(1)}(E)= \begin{cases}(1-\rho d t) \mathbb{E}_{E}\left[F_{0,1,1}^{(1)}(E+d E)\right] & , E<\epsilon_{0,1,1}^{(1)} \\ \Phi_{1,1}^{(1)}(E) & , E \geq \epsilon_{0,1,1}^{(1)}\end{cases}
$$

By expanding the first branch on the right-hand side of (A-1) using Itô's lemma, we obtain the differential equation for $F_{0,1,1}^{(1)}(E)$, which together with its solution is indicated in $(\mathrm{A}-2)$.

$$
\frac{1}{2} \sigma^{2} E^{2} F_{0,1,1}^{(1)^{\prime \prime}}(E)+\mu E F_{0,1,1}^{(1)^{\prime}}(E)-\rho F_{0,1,1}^{(1)}(E)=0 \Rightarrow F_{0,1,1}^{(1)}(E)=A_{0,1,1}^{(1)} E^{\beta_{1}}+C_{0,1,1}^{(1)} E^{\beta_{2}}
$$

Since $\beta_{2}<0$, we have $C_{0,1,1}^{(1)} E^{\beta_{2}} \rightarrow \infty$ as $E \rightarrow 0$. Hence, $C_{0,1,1}^{(1)}=0$ and $F_{0,1,1}^{(1)}(E)$ is indicated in $(4)$, where $A_{0,1,1}^{(1)}$ and $\epsilon_{0,1,1}^{(1)}$ are determined via the value-matching and smooth-pasting conditions between the two branches of (4). Similarly, in state $(0,0), A_{0,0}^{(1)}<0$ and $B_{0,0}^{(1)}>0$ are obtained via value-matching and smooth-pasting conditions between the two branches of (8) and are indicated in (A-3) and (A-4) respectively.

$$
\begin{aligned}
& A_{0,0}^{(1)}=\frac{\epsilon_{0,1,1}^{(1)-\delta_{1}}}{\delta_{2}-\delta_{1}}\left[\frac{\lambda\left(\delta_{2}-1\right) D_{1} \epsilon_{0,1,1}^{(1)}}{(\rho+\lambda-\mu)(\rho-\mu)}+\left(\beta_{1}-\delta_{2}\right) A_{0,1,1}^{(1)} \epsilon_{0,1,1}^{(1)^{\beta_{1}}}-\frac{\delta_{2} \lambda I_{1}}{\rho+\lambda}\right] \\
& B_{0,0}^{(1)}=\frac{\epsilon_{0,1,1}^{(1)}}{\delta_{1}-\delta_{2}}\left[\frac{\lambda\left(1-\delta_{1}\right) D_{1} \epsilon_{0,1,1}^{(1)}}{(\rho+\lambda-\mu)(\rho-\mu)}+\left(\delta_{1}-\beta_{1}\right) A_{0,1,1}^{(1)} \epsilon_{0,1,1}^{(1)^{\beta_{1}}}+\frac{\delta_{1} \lambda I_{1}}{\rho+\lambda}\right]
\end{aligned}
$$


Compulsive Strategy with $\mathrm{N}=2$

The value function in state $(1,2,2)$ is obtained following the same steps as in $(\mathrm{A}-1)$. In state $(1,1)$, the differential equation for $\Phi_{1,1}^{(2)}(E)$ is indicated in $(\mathrm{B}-1)$, and by solving $(\mathrm{B}-1)$ separately for $E<\epsilon_{1,2,2}^{(2)}$ and $E \geq \epsilon_{1,2,2}^{(2)}$ we obtain the expression for $\Phi_{1,1}^{(2)}(E)$, that is indicated in (13), where $A_{1,1}^{(2)}<0$ and $B_{1,1}^{(2)}>0$ are indicated in (B-2) and $(\mathrm{B}-3)$, respectively.

$$
\begin{aligned}
& \frac{1}{2} \sigma^{2} E^{2} \Phi_{1,1}^{(2)^{\prime \prime}}(E)+\mu E \Phi_{1,1}^{(2)^{\prime}}(E)-(\rho+\lambda) \Phi_{1,1}^{(2)}(E)+D_{1} E-\rho I_{1}+\lambda F_{1,2,2}^{(2)}(E)=0 \\
& A_{1,1}^{(2)}=\frac{\epsilon_{1,2,2}^{(2)}}{\delta_{2}-\delta_{1}}\left[\frac{\lambda\left(\delta_{2}-1\right)\left(D_{2}-D_{1}\right) \epsilon_{1,2,2}^{(2)}}{(\rho+\lambda-\mu)(\rho-\mu)}-\frac{\delta_{2} \lambda I_{2}}{\rho+\lambda}-\left(\delta_{2}-\beta_{1}\right) A_{1,2,2}^{(2)} \epsilon_{1,2,2}^{(2)^{\beta_{1}}}\right] \\
& B_{1,1}^{(2)}=\frac{\epsilon_{1,2,2}^{(2)^{-\delta_{2}}}}{\delta_{1}-\delta_{2}}\left[\frac{\lambda\left(1-\delta_{1}\right)\left(D_{2}-D_{1}\right) \epsilon_{1,2,2}^{(2)}}{(\rho+\lambda-\mu)(\rho-\mu)}+\frac{\delta_{1} \lambda I_{2}}{\rho+\lambda}+\left(\delta_{1}-\beta_{1}\right) A_{1,2,2}^{(2)} \epsilon_{1,2,2}^{(2)^{\beta_{1}}}\right]
\end{aligned}
$$

Next, the value function in state $(0,1,1), F_{0,1,1}^{(2)}(E)$, is indicated in $(14)$, where $A_{0,1,1}^{(2)}$ and $\epsilon_{0,1,1}^{(2)}$ are determined numerically via (B-4) and (B-5).

$$
\begin{aligned}
A_{0,1,1}^{(2)} \epsilon_{0,1,1}^{(2)^{\beta_{1}}} & =\Phi_{1,1}^{(2)}\left(\epsilon_{0,1,1}^{(2)}\right)+A_{1,2,2}^{(2)} \epsilon_{0,1,1}^{(2)^{\beta_{1}}}+A_{1,1}^{(2)} \epsilon_{0,1,1}^{(2)^{\delta_{1}}} \\
\beta_{1} A_{0,1,1}^{(2)} \epsilon_{0,1,1}^{(2)^{\beta_{1}}} & =\frac{D_{1} \epsilon_{0,1,1}^{(2)}}{\rho-\mu}+\beta_{1} A_{1,2,2}^{(2)} \epsilon_{0,1,1}^{(2)^{\beta_{1}}}+\delta_{1} A_{1,1} \epsilon_{0,1,1}^{(2)^{\delta_{1}}}
\end{aligned}
$$

Finally, the dynamics of the value function in state $(0,0)$ are described in $(\mathrm{B}-6)$, and the expression of $\Phi_{0,0}^{(2)}(E)$ is indicated in (17), where $A_{0,0}^{(2)}<0$ and $B_{0,0}^{(2)}>0$ are indicated in (B-7) and (B-8), respectively.

$$
\begin{aligned}
& \frac{1}{2} \sigma^{2} E^{2} \Phi_{0,0}^{(2)^{\prime \prime}}(E)+\mu E \Phi_{0,0}^{(2)^{\prime}}(E)-(\rho+\lambda) \Phi_{0,0}^{(2)}(E)+\lambda F_{0,1,1}^{(2)}(E)=0 \\
& A_{0,0}^{(2)}=\frac{\epsilon_{0,1,1}^{(2)}-\delta_{1}}{\delta_{2}-\delta_{1}}\left[\frac{\lambda\left(\delta_{2}-1\right) D_{1} \epsilon_{0,1,1}^{(2)}}{(\rho+\lambda-\mu)(\rho-\mu)}+\left(\beta_{1}-\delta_{2}\right) A_{0,1,1}^{(2)} \epsilon_{0,1,1}^{(2)^{\beta_{1}}}-\frac{\delta_{2} \lambda I_{1}}{\rho+\lambda}+\left(\delta_{2}-\beta_{1}\right) A_{1,2,2}^{(2)} \epsilon_{0,1,2}^{(2)^{\beta_{1}}}\right. \\
& \left.+\left(\delta_{2}-\delta_{1}\right) A_{1,1}^{(2)} \epsilon_{0,1,1}^{\delta_{1}}\right] \\
& B_{0,0}^{(2)}=\frac{\epsilon_{0,1,2}^{(2)}}{\delta_{1}-\delta_{2}}\left[\frac{\lambda\left(1-\delta_{1}\right) D_{1} \epsilon_{0,1,1}^{(2)}}{(\rho+\lambda-\mu)(\rho-\mu)}+\left(\delta_{1}-\beta_{1}\right) A_{0,1,1}^{(2)} \epsilon_{0,1,1}^{(2)^{\beta_{1}}}+\frac{\delta_{1} \lambda I_{1}}{\rho+\lambda}-\left(\delta_{1}-\beta_{1}\right) A_{1,2,2}^{(2)} \epsilon_{0,1,1}^{(2)^{\beta_{1}}}\right]
\end{aligned}
$$

Proposition $1 \forall \ell, m, n \in \mathbb{N}$ and $\forall \lambda \in \mathbb{R}^{+}, \Phi_{m, m}^{(n)}(E) \in\left[\Phi_{m, m}^{(m)}(E), F_{m, n, n}^{(n)}(E)\right)$.

Proof: The value of the option to invest in state $(m, n, n)$ is described in $(\mathrm{B}-9)$, while the value function in state $(m, m)$ under a compulsive strategy is described in $(\mathrm{B}-10)$. Notice that $\lambda=0 \Rightarrow \Phi_{m, m}^{(n)}(E)=\Phi_{m, m}^{(m)}(E)$, i.e., the value function at each state consists only of the value of the active project since no embedded options are available.

$$
\begin{aligned}
F_{m, n, n}^{(n)}(E) & =\Phi_{m, m}^{(m)}(E) \mathbb{1}_{\{m>0\}}+A_{m, n, n}^{(n)} E^{\beta_{1}}, \forall E<\epsilon_{m, n, n}^{(n)} \\
\Phi_{m, m}^{(n)} & (E)=\Phi_{m, m}^{(m)}(E) \mathbb{1}_{\{m>0\}}+A_{m, n, n}^{(n)} E^{\beta_{1}}+A_{m, m}^{(n)} E^{\delta_{1}}, \forall E<\epsilon_{m, n, n}^{(n)}
\end{aligned}
$$


Also, $\lambda \rightarrow \infty \Rightarrow E^{\delta_{1}} \rightarrow \infty$, and, since $A_{m, m}^{(n)}<0, A_{m, m}^{(n)} E^{\delta_{1}} \rightarrow-\infty$. Hence, $\lambda \rightarrow \infty \Rightarrow A_{m, m}^{(n)} \rightarrow 0$, and, thus, $\Phi_{m, m}^{(n)}(E) \in\left[\Phi_{m, m}^{(m)}(E), F_{m, n, n}^{(n)}(E)\right)$. Finally, (B-11) indicates the impact of $\lambda$ on the relative loss in $\Phi_{m, m}^{(n)}(E)$ due to technological uncertainty.

$$
\lim _{\lambda \rightarrow \infty} \frac{F_{m, n, n}^{(n)}(E)-\Phi_{m, m}^{(n)}(E)}{F_{m, n, n}^{(n)}(E)}=\lim _{\lambda \rightarrow \infty} \frac{-A_{m, m}^{(n)} E^{\delta_{1}}}{\Phi_{m, m}^{(m)}(E) \mathbb{1}_{\{\ell>0\}}+A_{m, n, n}^{(n)} E^{\beta_{1}}}=0
$$

Proposition 2 Under a compulsive strategy, $\forall \ell, m, n \in \mathbb{N}$ the impact of $\lambda$ on $\epsilon_{\ell, m, m}^{(n)}$ is non-monotonic.

Proof: By equating the $\mathrm{MB}$ of delaying investment to the $\mathrm{MC}$ in state $(\ell, m, m)$ we find that $\lambda$ impacts only the last term of the left- and right-hand side of (B-12).

$$
\frac{D_{m}}{\rho-\mu}+\frac{\beta_{1}}{\epsilon_{\ell, m, m}^{(n)}} I_{m}-\beta_{1} A_{m, m}^{(n)} \epsilon_{\ell, m, m}^{(n)^{\delta_{1}-1}}=\frac{\beta_{1} D_{m}}{\rho-\mu}-\delta_{1} A_{m, m}^{(n)} \epsilon_{\ell, m, m}^{(n)^{\delta_{1}-1}}
$$

Consequently, the overall impact of $\lambda$ on $\epsilon_{\ell, m, m}^{(n)}$ can be determined by its impact on these two terms. Therefore, we define the function $h(\lambda)=\delta_{1} A_{m, m}^{(n)}-\beta_{1} A_{m, m}^{(n)}$. As indicated in (B-13), $\beta_{1} A_{m, m}^{(n)}$ decreases by more than $\delta_{1} A_{m, m}^{(n)}$ when $\lambda$ is small, and, thus, the MB decreases by more than the MC, thereby lowering the optimal investment threshold.

$$
\lim _{\lambda \rightarrow 0} \frac{\partial h(\lambda)}{\partial \lambda}=\lim _{\lambda \rightarrow 0}\left\{\frac{\partial \delta_{1}}{\partial \lambda} A_{m, m}^{(n)}+\left(\delta_{1}-\beta_{1}\right) \frac{\partial A_{m, m}^{(n)}}{\partial \lambda}\right\}=-A_{m, n, n}^{(n)} \times \lim _{\lambda \rightarrow 0} \frac{\partial \delta_{1}}{\partial \lambda}<0
$$

At high values of $\lambda$, the MC decreases by more than the MB and the marginal value of delaying investment decreases, i.e., $\lim _{\lambda \rightarrow \infty} \frac{\partial h(\lambda)}{\partial \lambda}=\lim _{\lambda \rightarrow \infty}\left(\delta_{1}-\beta_{1}\right) \times \lim _{\lambda \rightarrow \infty} \frac{\partial A_{m, m}^{(n)}}{\partial \lambda}>0$

Proposition 3: A tradeoff between two technology versions $\ell$ and $n$ exists if $\frac{D_{n}}{\sum_{i=1}^{n} I_{i}}<\frac{D_{\ell}}{\sum_{i=1}^{\ell} I_{i}}$, $\forall \ell, n$ with $\ell<n$. Proof: We denote by $\varepsilon$ the indifference point, i.e., the output price at which the NPVs of the two projects intersect. For simplicity, we will assume that there is no technological uncertainty, and, thus, ignore any embedded options implied by the arrival of new technology versions. In addition, we assume that $n=\ell+1$ so that $\ell$ and $n$ represent two subsequent technology versions. Thus, the expression of $\varepsilon$ is indicated in (B-14).

$$
\begin{aligned}
\Phi_{\ell, \ell}^{(\ell)}(\varepsilon)=\Phi_{n, n}^{(n)}(\varepsilon) & \Rightarrow \frac{D_{\ell} \varepsilon}{\rho-\mu}-\sum_{i=1}^{\ell} I_{i}=\frac{D_{n} \varepsilon}{\rho-\mu}-\sum_{i=1}^{n} I_{i} \\
& \Rightarrow \varepsilon=\frac{(\rho-\mu) I_{n}}{D_{n}-D_{\ell}}
\end{aligned}
$$

In order to have a tradeoff between the two projects, their value functions at the indifference point $\varepsilon$ must be positive. Otherwise, only investment in the new technology version is optimal. Consequently, the condition that must be satisfied for a tradeoff to exist is indicated in (B-15).

$$
\Phi_{n, n}^{(n)}(\varepsilon)>0 \Leftrightarrow \frac{D_{n}}{\sum_{i=1}^{n} I_{i}}<\frac{D_{\ell}}{\sum_{i=1}^{\ell} I_{i}}
$$


Proposition 4: Under a a leapfrog or laggard strategy, $\forall \ell, m, n \in \mathbb{N}$ we have $\epsilon_{\ell, m, m}^{(n)}<\underline{\epsilon}_{\ell, m, n}^{(n)} \forall \lambda \in(0,+\infty)$, whereas $\lambda=0 \Rightarrow \epsilon_{\ell, m, m}^{(n)}=\underline{\epsilon}_{\ell, m, n}^{(n)}$ and $\lambda \rightarrow \infty \Rightarrow \epsilon_{\ell, m, m}^{(n)} \rightarrow \underline{\epsilon}_{\ell, m, n}^{(n)}$.

Proof: First, we rewrite the value of the option to invest in state $(\ell, m, n)$ as in $(\mathrm{B}-16)$

$$
F_{\ell, m, n}^{(n)}(E)=\max _{\underline{\epsilon}_{\ell, m, n}^{(n)}>E}\left(\frac{E}{\underline{\epsilon}_{\ell, m, n}^{(n)}}\right)^{\beta_{1}}\left[\frac{\epsilon_{\ell, m, n}^{(n)} D_{m}}{\rho-\mu}-I_{m}+A_{m, n, n}^{(n)} \underline{\epsilon}_{\ell, m, n}^{(n)^{\beta_{1}}}\right]
$$

and then apply first-order necessary conditions to (B-16) in order obtain the expression for $\underline{\epsilon}_{\ell, m, n}^{(n)}$, which is indicated in (B-17). Notice that $\epsilon_{\ell, m, m}^{(n)}=\underline{\epsilon}_{\ell, m, n}^{(n)}$ for $\lambda=0$, whereas $\lambda \rightarrow \infty$ implies that the likelihood of at least one innovation occurring converges to one, and, therefore, we have $\epsilon_{\ell, m, m}^{(n)} \rightarrow \underline{\epsilon}_{\ell, m, n}^{(n)}$. Finally, $\epsilon_{\ell, m, m}^{(n)}<\underline{\epsilon}_{\ell, m, n}^{(n)}$ follows from Proposition 2.

$$
\underline{\epsilon}_{\ell, m, n}^{(n)}=\frac{\beta_{1}}{\beta_{1}-1} \frac{I_{m}(\rho-\mu)}{D_{m}}
$$

Proposition 5: $\forall E \in\left(0, \bar{\epsilon}_{0,1,2}^{(2)}\right]$, if the investment region in state $(0,1 \vee 2)$ is dichotomous, then the compulsive strategy dominates the leapfrog/laggard strategy $\forall \lambda \in \mathbb{R}^{+}$.

Proof: If the investment region in state $(0,1 \vee 2)$ is not dichotomous, then it is optimal to wait until $E=\epsilon_{0,2,2}^{(2)}$ and then invest in the second version (Dixit, 1993). However, if the investment region is dichotomous and $E \in\left(0, \bar{\epsilon}_{0,1,2}^{(2)}\right]$, then the expected NPV from investment in the first version of the technology under a compulsive strategy is indicated in (13). Although the payoff under a laggard strategy is the same, it is, nevertheless, conditional on the arrival of the second version, and, therefore, is lower compared to the immediate profit from a compulsive strategy.

Proposition 6: $\exists \underline{\lambda}, \bar{\lambda} \in \mathbb{R}^{+}$with $\underline{\lambda} \leq \bar{\lambda}$ :

$\mathrm{i}: \forall \lambda>\underline{\lambda}, \exists \mathcal{B} \subseteq\left(\epsilon_{0,2,2}^{(2)}, \infty\right): \forall E \in \mathcal{B}$, the leapfrog strategy dominates

ii : $\forall \lambda>\bar{\lambda}, \exists \mathcal{A} \subset\left[\bar{\epsilon}_{0,1,2}^{(2)}, \epsilon_{0,2,2}^{(2)}\right): \forall E \in \mathcal{A}$, the leapfrog/laggard strategy dominates

Proof: (i) Under a compulsive strategy and for $E \geq \epsilon_{0,2,2}^{(2)}$, we have $\lambda=0 \Rightarrow F_{0,1,1}^{(2)}(E)=\Phi_{1,1}^{(1)}(E)$ and $\lim _{\lambda \rightarrow \infty} F_{0,1,1}^{(2)}(E)=\Phi_{1,1}^{(1)}(E)+A_{1,2,2}^{(2)} E^{\beta_{1}}$. Also, according to Proposition 2, $\lambda \nearrow \Rightarrow\left|A_{1,1}^{(2)}\right| \searrow$ which implies that $\frac{\partial F_{0,1,1}^{(2)}(E)}{\partial \lambda}>0 \forall \lambda \in \mathbb{R}^{+}$. Additionally, under a leapfrog strategy and for $E \geq \epsilon_{0,2,2}^{(2)}$, we have $\lambda=0 \Rightarrow F_{0,1,1}^{(2)}(E)=0$ and $\lim _{\lambda \rightarrow \infty} F_{0,1,1}^{(2)}(E)=\frac{D_{2} E}{\rho-\mu}-I_{2}$ Hence, if $\lambda$ is low, e.g., $\lambda=0$, then the compulsive strategy dominates since $\Phi_{1,1}^{(2)}(E) \geq 0, \forall E \geq 0$. By contrast, as $\lambda \rightarrow \infty$, the leapfrog strategy dominates. Indeed, if $\varepsilon: \Phi_{\overline{2,2}}^{(2)}(\varepsilon)=F_{1,2,2}^{(2)}(\varepsilon)$, then $\varepsilon<\epsilon_{0,2,2}^{(2)}$. If we set $\mathbb{L}=\left\{\lambda \mid \exists \mathcal{B} \subseteq\left(\epsilon_{0,2,2}^{(2)}, \infty\right):\right.$ leapfrog dominates $\left.\forall E \in \mathcal{B}\right\}$, then $\exists \underline{\lambda} \in \mathbb{R}^{+}: \underline{\lambda}=\min \lambda \in \mathbb{L}$ and

$$
\frac{\underline{\lambda} D_{2} E}{(\rho+\underline{\lambda}-\mu)(\rho-\mu)}-\frac{\underline{\lambda} I_{2}}{\rho+\underline{\lambda}}+J_{0,1,1}^{(2)} E^{\delta_{2}}=\Phi_{1,1}^{(2)}(E)+A_{1,2,2}^{(2)} E^{\beta_{1}}+A_{1,1}^{(2)} E^{\delta_{1}}
$$


while, $\forall \lambda>\underline{\lambda}, \exists \mathcal{B} \subseteq\left(\epsilon_{0,2,2}^{(2)}, \infty\right)$ such that the leapfrog strategy dominates $\forall E \in \mathcal{B}$.

(ii) The derivation is similar to (i) and follows from the convexity of $F_{0,1,1}^{(2)}(E)$ and the value-matching and smooth-pasting conditions that ensure that $F_{0,1,1}^{(2)}(E)$ is $\mathcal{C}^{1}, \forall E>0$.

\section{References}

1. Balcer, Y and S Lippman (1984), "Technological Expectations and Adoption of Improved Technology," Journal of Economic Theory, 34: 292-318.

2. Boomsma, TK, N Meade, and SE Fleten (2012), "Renewable Energy Investments under Different Support Schemes: A Real Options Approach," European Journal of Operational Research, 220(1): 225-237.

3. Chronopoulos, M, B De Reyck, and A Siddiqui (2013), "The Value of Capacity Sizing under Risk Aversion and Operational Flexibility," IEEE Trasactions on Engineering Management 60(2): 272-288.

4. Décamps, JP, T Mariotti, and S Villeneuve (2006), "Irreversible Investment in Alternative Projects," Economic Theory, 28: $425-448$.

5. Dixit, AK (1993), "Choosing Among Alternative Discrete Investment Projects under Uncertainty," Economics Letters, 41: $265-268$.

6. Dixit, AK and RS Pindyck (1994), Investment under Uncertainty, Princeton University Press, Princeton, NJ, USA.

7. Doraszelski, U (2001), "The Net Present Value Method versus the Option Value of Waiting: A Note on Farzin, Huisman and Kort (1998)," Journal of Economic Dynamics \&3 Control, 25: 1109-1115.

8. Farzin, Y, KJM Huisman, and PM Kort (1998), "Optimal Timing of Technology Adoption," Journal of Economic Dynamics and Control, 22: 779-799.

9. Financial Times (2012a), "Burden of Obsolescence Becomes more Acute," 6 May.

10. Financial Times (2012b), "Vestas a Victim of its own Propaganda," 1 March.

11. Franklin, SL (2014), "Investment Decisions in Mobile Telecommunications Networks Applying Real Options," Annals of Operations Research, DOI 10.1007/s10479-014-1672-9.

12. Gollier, C, D Proult, F Thais, and G Walgenwitz (2005), "Choice of Nuclear Power Investments under Price Uncertainty: Valuing Modularity," Energy Economics, 27: 667-685.

13. Grenadier, SR and AM Weiss (1997), "Investment in Technological Innovations: An Option Pricing Approach," Journal of Financial Economics, 44: 397-416.

14. Huisman, K and PM Kort (2004), "Strategic Technology Adoption Taking into Account Future Technological Improvements: A Real Options Approach", European Journal of Operational Research, 159: 705-728.

15. International Business Times (2014), "Apple iPhone 5s Lacks Consumer Interest New Report Says," 21 May.

16. Jensen, P, P Morthorst, S Skriver, M Rasmussen, H Larsen, L Henrik Hansen, P Nielsen, and J Lemming (2002), Økonomi for Vindmøller $i$ Danmark, Annual Report No. 1247, Technical University of Denmark, Denmark.

17. Kauffman, RJ and X Li (2005), "Technology Competition and Optimal Investment Timing: A Real Options Perspective," IEEE Transactions on Engineering Management, 52(1):15-29.

18. Kort, PM, P Murto, and P Grzegorz (2010), "Uncertainty and Stepwise Investment," European Journal of Operational Research, 202(1): 196-203.

19. MacGillivray, A, H Jeffrey, M Winskel, and I Bryden (2014), "Innovation and Cost Reduction for Marine Renewable Energy: A Learning Investment Sensitivity Analysis," Technological Forecasting and Social Change, 87: 108-124.

20. Majd, S and RS Pindyck (1987), "Time to Build, Option Value, and Investment Decisions," Journal of Financial Economics, 18: $7-27$.

21. Malchow-Møller, N and BJ Thorsen (2005), "Repeated Real Options: Optimal Investment Behaviour and a Good Rule of Thumb," Journal of Economic Dynamics \& Control, 29: 1025-1041. 
22. Mauritzen, J (2014), "Scrapping a Wind Turbine: Policy Changes, Scrapping Incentives and why Wind Turbines in Good Locations get Scrapped First," The Energy Journal, 35(2): 157-181.

23. Miltersen, RK and E Schwartz (2007), "Real Options with Uncertainty Maturity and Competition," NBER working paper series.

24. Siddiqui, A and S-E Fleten (2010), "How to Proceed with Competing Alternative Energy Technologies: A Real Options Analysis," Energy Economics, 32: 817-830.

25. The Economist (2009), "Planned Obsolescence," 23 March.

26. Wind Power (2012), "Upgrade old Turbines to their Full Potential," 1 December. 